\title{
MTG: resolution enhancement for MW measurements from geostationary orbits
}

\author{
S. Dietrich, F. Di Paola, and B. Bizzarri \\ Istituto di Scienze dell'Atmosfera e del Clima, Consiglio Nazionale delle Ricerche, Roma, Italy \\ Received: 31 October 2005 - Revised: 22 January 2006 - Accepted: 22 February 2006 - Published: 18 April 2006
}

\begin{abstract}
The purpose of this study is to develop and evaluate image processing techniques that improve the spatial resolution of the channels already selected in the preliminary studies for "Geostationary Observatory for Microwave Atmospheric Soundings (GOMAS)". Reference high resolution multifrequency brightness temperatures scenarios have been derived by applying radiative transfer calculation to the spatially and microphysically detailed output of meteorological events simulated by the University of Wisconsin - Non-hydrostatic Model System. Two approaches, Wiener filter and SIR algorithm, have been applied to low frequency channels to enhance the resolution of antenna temperatures, exploiting the oversampling available for GOMAS channels observational strategy. Quite similar improvements have been obtained by applying the two techniques, even if SIR algorithm has provided generally better performances at computation time's expense.
\end{abstract}

\section{Introduction}

The follow-on studies of the post-MSG consultation process considered the technical possibility of measuring precipitation from Meteosat Third Generation (MTG) by using microwaves in addition to what is possible to infer from VIS/IR imagery. The main obstacle is represented by the low spatial resolution achievable by microwave (MW) imagery from Geosynchronous Earth Orbit (GEO). This implies that possible convective cells appear too smooth, making it impossible to exactly identify the precipitating region and to estimate the rainfall intensity. In order to improve spatial resolution, several post-processing deconvolution techniques are under evaluation in the frame of the EUMETSAT project "Simulations and User Requirements Review for Precipitating Clouds from Geostationary orbits in $\mathrm{Mm} / \mathrm{Submm}$ Bands (GeoRain)". In this study we use few channels among the 40 ones proposed for "Geostationary Observatory for for Microwave Atmospheric Soundings (GOMAS)". The selected channels (Table 1) are mainly the most transparent in each band $[50.300 \mathrm{GHz},(118.750 \pm 5.000)$ $\mathrm{GHz},(183.310 \pm 17.000) \mathrm{GHz},(380.197 \pm 18.000) \mathrm{GHz}$ and $(424.763 \pm 4.000) \mathrm{GHz}$. The selected channels of the 380 and $425 \mathrm{GHz}$ band are blind to the lower troposphere due to the water vapour continuum, having the weighting functions peaking approximately at $5 \mathrm{~km}$. Then we include also the channels $[53.845 \mathrm{GHz},(118.750 \pm 2.100) \mathrm{GHz}$ and $(183.310 \pm 5.000) \mathrm{GHz}]$ since they peak at $5 \mathrm{~km}$ too. Their spatial resolution characteristics are representative for all the GOMAS channels.

The principles of resolution enhancement of MW images have been stated long ago (see, for instance, Buck and Gustincic, 1967; Ulaby et al., 1981; Skou, 1989). However, practical applications are lacking because most instruments so far flown have been designed under constraints that led to far-from-optimal sampling. Typical example is the Special Sensor Microwave/Imager (SSM/I), which is under-sampled at the highest frequency.

The deconvolution technique (Poe, 1990; Farrar and Smith, 1992) can be applied when the image is heavily oversampled, at least with Nyquist (100\% oversampling, i.e. double sampling rate in respect of what would be needed to have adjacent Instantaneous

Field Of View - IFOV's). Since all channels in GOMAS are sampled at $10 \mathrm{~km}$ intervals, this condition applies to $54 \mathrm{GHz}(81 \mathrm{~km} \mathrm{IFOV}), 118 \mathrm{GHz}(37 \mathrm{~km})$ and $183 \mathrm{GHz}$ $(24 \mathrm{~km})$. Another technique that can be used for heavily oversampled channels is the the Scatterometer Image Reconstruction (SIR) algorithm, based on an iterative processing, which will be explained later.

The 380 and $425 \mathrm{GHz}$ bands are not oversampled. In this case, it is still possible to improve the details in the image by exploiting the bi-linear interpolation principle. 
Table 1. GOMAS channels to be simulated. The integration time corresponding to one $10-\mathrm{km}$ pixel is $6 \mathrm{~ms}$.

\begin{tabular}{cccccc}
\hline$v(\mathrm{GHz})$ & $\Delta v(\mathrm{MHz})$ & IFOV $(\mathrm{km})$ & Pixel $(\mathrm{km})$ & $N E \Delta T$ at pixel level $(\mathrm{K})$ & Peak of weighting function \\
\hline 53.845 & 190 & 81 & 10 & 0.48 & $5 \mathrm{~km}$ \\
50.300 & 180 & 81 & 10 & 0.48 & Surface \\
$118.750 \pm 2.100$ & 800 & 37 & 10 & 0.33 & $5 \mathrm{~km}$ \\
$118.750 \pm 5.000$ & 2000 & 37 & 10 & 0.21 & Surface \\
$183.310 \pm 5.000$ & 2000 & 24 & 10 & 0.34 & $5 \mathrm{~km}$ \\
$183.310 \pm 17.000$ & 4000 & 24 & 10 & 0.36 & Surface \\
$380.197 \pm 18.000$ & 2000 & 12 & 10 & 0.72 & $6 \mathrm{~km}$ \\
$424.763 \pm 4.000$ & 1000 & 10 & 10 & 1.02 & $5 \mathrm{~km}$ \\
\hline
\end{tabular}

\section{Experimental settings}

Advanced numerical models can provide a detailed description of cloud microphysics, making it possible the calculation of brightness temperature, at different frequencies over wide geographic areas, with the resolution of the cloud model. So we used high resolution scenarios built up from the microphysically detailed output of the "University of Wisconsin Non-hydrostatic Model System" (UW-NMS) (Tripoli, 1992) followed by the Radiative Transfer Model (Bauer et al., 1998), appropriate to millimetre-submillimetre wave channels. Specifically, two simulated events have been used in this paper: a Baltic light snowfall occurred over Gotland island from 12:00 UTC 11 January to 06:00 UTC 12 January 2003, and a winter frontal system over Piemonte region (Italy) from 12:00 UTC 24 November to 06:00 UTC 26 November, 2002. The resulting simulated MW images have been provided in $200 \times 200$ pixel regular grid for Gotland and $141 \times 261$ pixel regular grid for Piemonte case. Pixel data, corresponding and spaced at $2.34 \mathrm{~km}$, derive directly from the corresponding model resolution cell. Considering simulated scenarios as "possible" meteorological occurrences, the obtained multi-frequency brightness temperature $(T B)$ maps represent for us the archive of reference truth for validating the resolution enhancement studies.

A microwave image aims at reconstructing the brightness temperature distribution of a given scenario. By means of the antenna beam scanning it is possible to record the brightness temperature angular distribution as seen from the radiometer system. Then it is practical to consider the antenna system (antenna + scanning system) as a transducer that operates on the brightness temperature distribution. The chain to generate the Antenna Temperature (TA) is described in Fig. 1.

The observed brightness distribution can be considered as the convolution of the antenna directivity function with the true brightness distribution.

The GOMAS instrument is expected to sample at about $10 \mathrm{~km}$. In order to reproduce this kind of observational product we have to consider available not all of the pixels, but we must extract 1 pixel every $16(4 \times 4)$, from the matrices containing the result of the convolution process. This operation represents a sub-sampling and, in order to not introduce aliasing or more generally to distort the Fourier transform of the image, we have to check to be still in the sampling theorem, that means to sample at a frequency double of the higher frequency included in the signal. This condition has been verified by analysing the spatial frequency spectrum. Finally, in order to obtain the $T A$, we have simulated the radiometric noise by a random generation of noise values having gaussian distribution with $\sigma$ equal to the expected $N E \Delta T$ of the specific frequency channel (see Table 1). In particular, the $N E \Delta T$ values in the Table 1 depend on the status of the art and are deduced from the formula:

$T_{\text {sys }}=N E \Delta T \cdot \Delta v^{0.5} \cdot t^{0.5}$

where $T_{s y s}$ is the temperature of the system, $v$ is the band width and $t$ is the integration time. These values are added to the result of convolution, finally producing the map that we will consider the GOMAS-like observation. Figure 2 is an example of the level of degradation for the low frequency channel at $50.35 \mathrm{GHz}$ for the Piemonte case.

\section{Resolution enhancement}

It is necessary to distinguish two cases for which we treat images in a different manner:

1. case of oversampled image, at least with Nyquist ( $100 \%$ oversampling, i.e. double sampling rate in respect of what would be needed to have adjacent IFOV's). Since all channels in GOMAS are sampled at $10 \mathrm{~km}$ intervals, this condition applies to $54 \mathrm{GHz}(81 \mathrm{~km}$ IFOV), $118 \mathrm{GHz}(37 \mathrm{~km})$ and $183 \mathrm{GHz}(24 \mathrm{~km})$.

2. case of not oversampled image. This condition applies to $380 \mathrm{GHz}(12 \mathrm{~km} \mathrm{IFOV})$ and $425 \mathrm{GHz}(10 \mathrm{~km})$.

\subsection{Case of oversampled image: Wiener filter}

For the oversampled image it is possible to apply the deconvolution technique by means of Wiener filter. In order to apply this technique it is necessary to operate in the domain of the spatial frequencies. The reconstructed image $T B^{\prime}$ is 


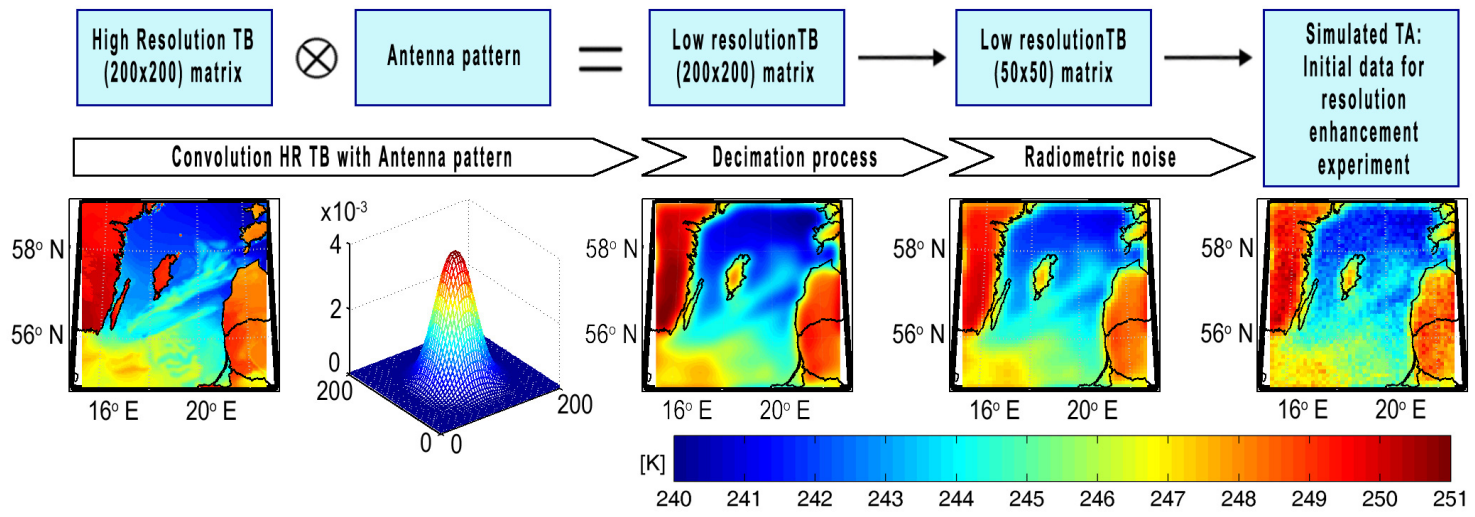

Fig. 1. Experimental setting described through Gotland region images at (118.750 \pm 2.100$) \mathrm{GHz}$.

a)

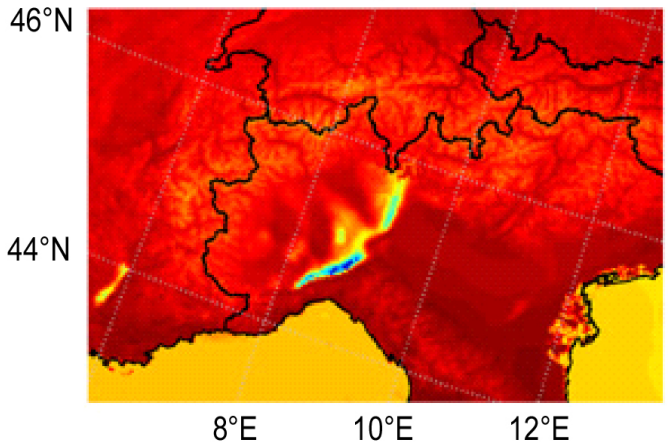

b)

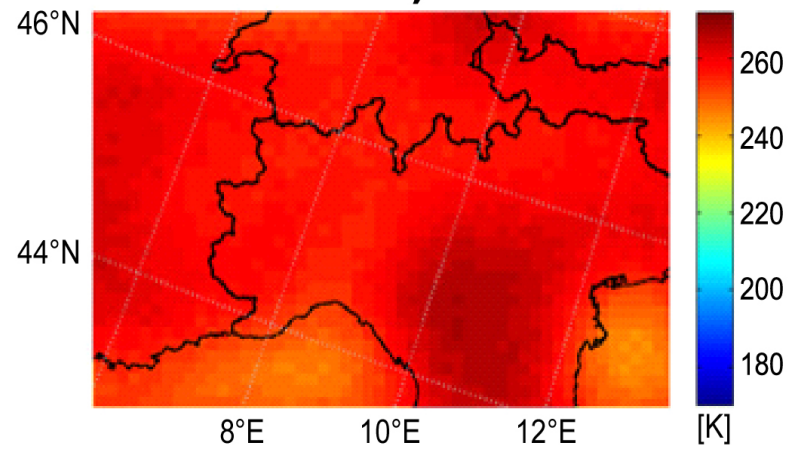

Fig. 2. Brightness temperature - Piemonte case at 50.35 GHz: (a) simulated TB (high resolution), (b) $T A$ (low resolution)

obtained by the inverse Fourier transform of the product between the Wiener filter $(W)$ and the Fourier transform of the antenna temperature pattern $(T A)$.

$$
W(u, v)=\frac{P_{T B T B}(u, v) H(u, v)^{*}}{P_{T B T B}(u, v)|H(u, v)|^{2}+P_{N N}(u, v)}
$$

where the $(u, v)$ denotes the spatial frequency coordinates, $P_{T B T B}(u, v)$ the power spectrum of $T B, P_{N N}(u, v)$ the power spectrum of radiometric noise, $H(u, v)$ the Fourier transform of the antenna pattern and the symbol * denotes the complex conjugate.

In order to implement the Wiener filter in practice we have to estimate the power spectrum of the original image and the additive noise. For white additive noise the power spectrum is equal to the variance of the noise:

$$
P_{N N}(u, v)=\sigma^{2}
$$

To estimate the power spectrum of the original image many methods can be used. Generally, periodogramm approach, a large number of observations represents the basic statistical information to derive the power spectrum estimate of original scenario. Unfortunately this method is not applicable in our case.
Another estimate which leads to a cascade implementation of the inverse filtering and the noise smoothing is:

$$
P_{T B T B}(u, v)=\frac{P_{T A T A}(u, v)-P_{N N}(u, v)}{|H(u, v)|^{2}}
$$

which is a straightforward result of the fact:

$$
P_{T A T A}(u, v)=P_{T B T B}(u, v)|H(u, v)|^{2}+P_{N N}(u, v)
$$

The disadvantage of this implementation is that $H(u, v)$ matrix can be singular: in this cases (Jain, 1989) we have to use a threshold value to remove the singularity. Combining above equation we obtain the final formula for the Wiener filter:

$$
\begin{aligned}
& W(u, v)=\frac{P_{T A T A}(u, v)-\sigma^{2}}{H(u, v) \cdot P_{T A T A}(u, v)} \\
& T B^{\prime}(x, y)=I F T[W(u, v) T A(u, v)]
\end{aligned}
$$

where the IFT[] denote the Inverse Fourier Transform.

\subsection{Case of oversampled image: SIR algorithm}

The Scatterometer Image Reconstruction (SIR) algorithm was originally designed to produce multivariate scatterometer images (Long et al., 1993). Then this algorithm has been 


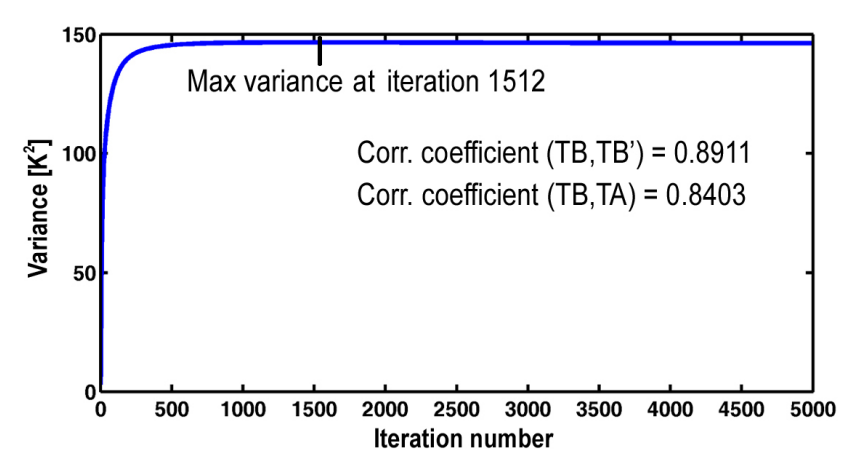

Fig. 3. Variance of reconstructed image vs Iteration number for SIR algorithm - Gotland case @ $50.300 \mathrm{GHz}$.

also adapted to work with radiometric measurements (Long et al., 1998).

It produces radiometric images by applying an iterative procedure to $T A(x, y)$. The procedure is nonlinear and the obtainable resolution enhancement depends on the antenna pattern dimension, shape, and measurement overlap.

In implementing the SIR algorithm, an initial guess for the $T B$ ' image is first made: in our case the $T A$ map. A predicted value (forward projection) of each measurement is calculated from a current estimate of $T B^{\prime}$ ' and is compared to the measurement. A scale factor is then computed as the ratio of the measurement to the forward projection. An update term is computed for each pixel in the measurement cell by multiplying by the scale factor. Between iterations, each pixel in the image is updated by averaging the update terms for the pixel. As the process iterates, the scale factors approach unity and all of the forward projections match the measurements. When noise is present in the measurements, however, the scale factors may not always converge to unity. In this case, the algorithm attempts to balance the various scale factors for each measurement and pixel using maximum entropy. In SIR, the scale factors calculated for each forward projection are damped by taking the square root. In addition, the update terms are computed in a manner that limits the amount of change for a single update. These steps tend to reduce the sensitivity of the update terms to noise.

Defining $T A_{i}$ the $i$-th pixel of antenna temperature, $T B^{, k}$ the $j$-th pixel of reconstructed brightness temperature at the $k$-th iteration, and $h_{i j}$ the correspondent weighting function, the forward projection $f_{i}^{k}$ to the $k$-th iteration is calculated as:

$f_{i}^{k}=\frac{1}{q_{i}} \sum_{n=1}^{M} h_{i} T B_{n}^{\prime k}$

$q_{i}=\sum_{n=1}^{M} h_{i n}$

where $M$ is the number of pixels in the image. The scale factor $d_{i}^{k}$ is then computed as:

$d_{i}^{k}=\left[\frac{T A_{i}}{f_{i}^{k}}\right]^{\gamma}$

where $\gamma$ is an adjustable parameter. The optimal result of Spatial Resolution Enhancement of SSM/I Data is with $\gamma=0.5$.

The nonlinear update term $u_{i j}^{k}$ is then computed, according to:

$u_{i j}^{k}= \begin{cases}{\left[\frac{1}{2 f_{i}^{k}}\left(1-\frac{1}{d_{i}^{k}}\right)+\frac{1}{T B_{j}^{\prime k} d_{i}^{k}}\right]^{-1} \quad \text { if } d_{i}^{k} \geq 1} \\ {\left[\frac{1}{2} f_{i}^{k}\left(1-d_{i}^{k}\right)+T B_{j}^{\prime k} d_{i}^{k}\right]} & \text { if } d_{i}^{k}<1\end{cases}$

After the entire data set has been processed, each pixel estimate $T B_{j}{ }_{j}$ is updated by computing a weighted average of the update terms, i.e.:

$T B_{j}^{\prime k+1}=\frac{1}{q_{j}} \sum_{i=1}^{M} h_{i j} u_{i j}^{k}$

$q_{j}=\sum_{j=1}^{M} h_{i j}$

Generally, this set of equations is iterated over $k$ for $N$ iterations until the scale factors approach unity. As a matter of fact, we have seen that the accuracy of the algorithm at first increases with the iteration number, but then may slightly decrease as the iteration continues. This is a common problem with algebraic reconstruction algorithms and is the result of the noise amplification. Operationally, we have found that the optimal criterion to select the iteration number can be based upon the evaluation of the variance of the reconstructed image, stopping the iteration as soon as it reaches the maximum value (see for example Fig. 3 for Gotland case at $50.300 \mathrm{GHz}$ ).

\subsection{Case of not oversampled image: bilinear interpolation}

Aiming at improving the correlation between $T A$ and $T B$ for the not oversampled channels ( 380 and $425 \mathrm{GHz}$ ), we have simply applied the bilinear interpolation to the $T A$ rectangular grid. Bilinear interpolation involves linear interpolation between adjacent points along one axis of the grid, followed by the linear interpolation along the other one. This interpolation is equivalent to the convolution of the gridded data (with zeros filled in between the original samples) with an interpolation function shaped like a square pyramid with drooping walls; the base of the pyramid is double of the sampling rate.

\section{Results and conclusions}

Gotland and Piemonte cases can be used to show the effect of Wiener and SIR algorithms when different signal to noise ratios (SNR) are encountered in TA. For Gotland, 
TB

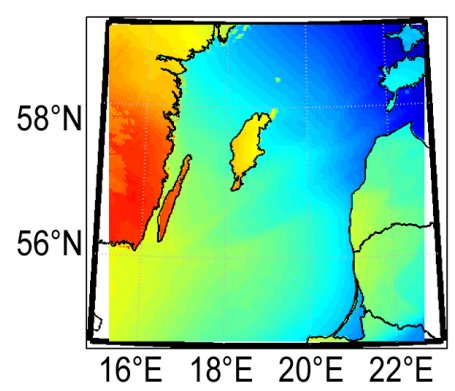

TA

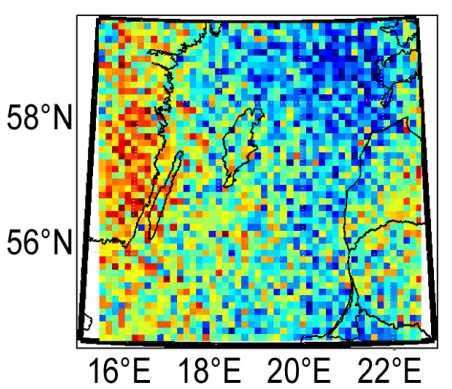

Reconstructed TB

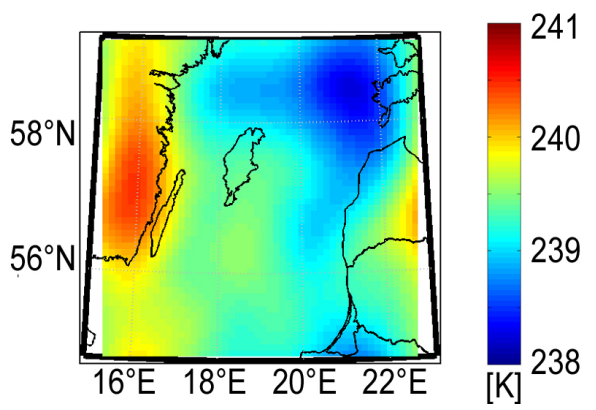

Fig. 4. Gotland region at $53.845 \mathrm{GHz}$.

TB

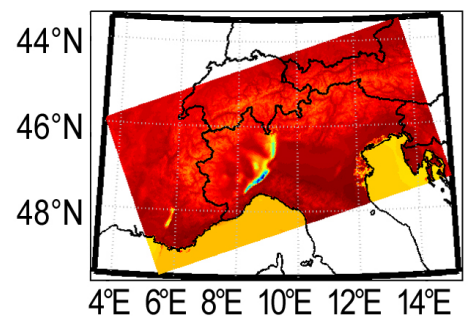

TA

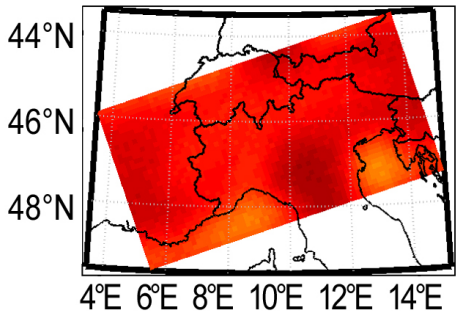

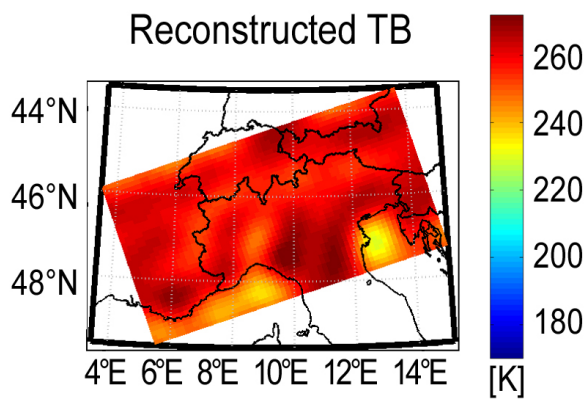

Fig. 5. Piemonte region at $50.300 \mathrm{GHz}$.

the thin MW dynamic range in reference $T B$ (Fig. 4, left panel) is overcome by the noise figure added for generating $T A$ (Fig. 4, central panel). Under this condition, it is clear that reconstructed $T B$ images are qualitatively better than $T A$ mainly due to the denoising effect produced by the employed techniques. In our study, the above situation occurs at low frequency channels $(54 \mathrm{GHz}, 118 \mathrm{GHz}$ and $183 \mathrm{GHz})$ if the SNR of $T A$ is lower than about 5 . On the other hand, at the same frequencies, if the SNR of $T A$ is at least $\sim 5$, like in the Piemonte case, the proposed techniques provide clear resolution enhancement effects. For example, the left panel of Fig. 5 shows a reference $T B$ map including a cloud structure, extended approximately $120 \times 10 \mathrm{~km}$, which is not visible in $T A$ map (Fig. 5, central panel) but whose presence can be recognized in the reconstructed $T B$ map (Fig. 5, right panel). Similarly, the resolution enhancement effect is also recognizable in Fig. 6, for example focusing on the contours of the coasts of the Gotland island. However, the task of feature distinction is only one of the possible intents of image processing. In order to objectively quantify the performance of the techniques in reproducing the reference $T B$ images starting from noisy $T A$ maps, we have expressed the comparative results in Tables 2 and 3 in terms of correlation coefficient between the images. Under this perspective, the SIR algorithm generally produces a better image reconstruction than Wiener filter, even if the high optimal number of requested iterations largely increase the computation time. Nevertheless, both methods exploit remarkably the information represented by the large oversampling when applied to low frequency channels.
Table 2. Results for Gotland Region.

\begin{tabular}{ccll}
\hline$v(\mathrm{GHz})$ & Corr. coeff. $(T B, T A)$ & \multicolumn{2}{l}{ Corr. coeff. $\left(T B, T B^{\prime}\right)$} \\
\hline 53.845 & 0.58 & Wiener & 0.89 \\
& & SIR & 0.90 \\
50.300 & 0.84 & Wiener & 0.87 \\
& & SIR & 0.89 \\
$118.750 \pm 2.100$ & 0.89 & Wiener & 0.91 \\
& & SIR & 0.92 \\
$118.750 \pm 5.000$ & 0.89 & Wiener & 0.92 \\
& & SIR & 0.93 \\
$183.310 \pm 5.000$ & 0.68 & Wiener & 0.82 \\
& & SIR & 0.89 \\
$183.310 \pm 17.000$ & 0.93 & Wiener & 0.94 \\
& & SIR & 0.94 \\
$380.197 \pm 18.000$ & \multirow{2}{*}{0.28} & Bilinear & 0.36 \\
$424.763 \pm 4.000$ & \multirow{2}{*}{0.18} & Interp. \\
& & Bilinear & 0.24 \\
& & Interp. \\
\hline
\end{tabular}

At high frequency channels $(380 \mathrm{GHz}$ and $425 \mathrm{GHz}$ ) we have very high SNR and the bilinear interpolation increases the correlation. Although we cannot properly talk about a "resolution enhancement" effect, since the interpolation do not add information to the available $T A$ data, the image details appear clearly more defined (see for example Fig. 7). 
TB

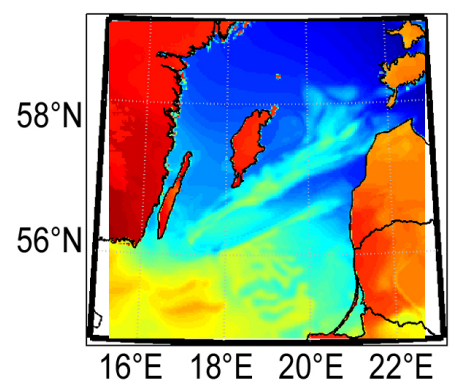

TA

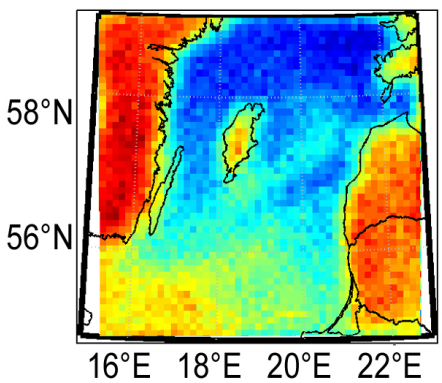

Reconstructed TB

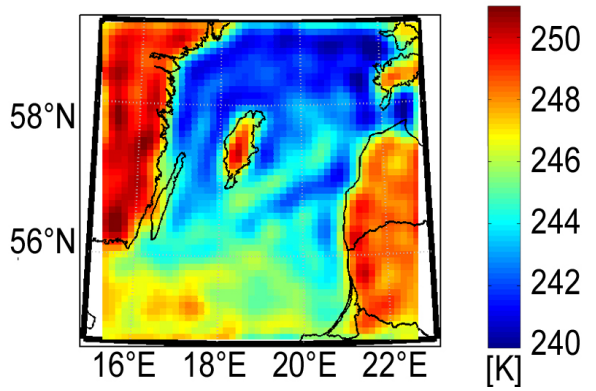

Fig. 6. Gotland region at $(118.750 \pm 2.100) \mathrm{GHz}$.

TB

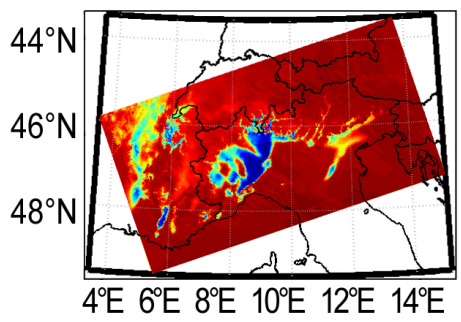

TA

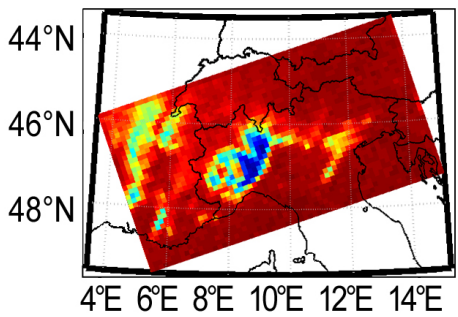

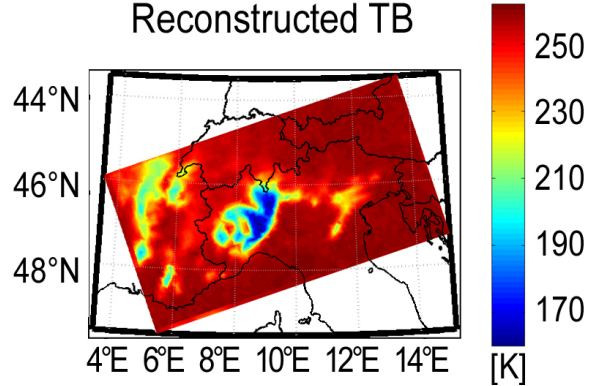

Fig. 7. Piemonte region at $(380.197 \pm 18.000) \mathrm{GHz}$.

Table 3. Results for Piemonte Region.

\begin{tabular}{ccll}
\hline$v(\mathrm{GHz})$ & Corr. coeff. $(T B, T A)$ & \multicolumn{2}{l}{ Corr. coeff. $\left(T B, T B^{\prime}\right)$} \\
\hline 53.845 & 0.55 & Wiener & 0.61 \\
& & SIR & 0.61 \\
50.300 & 0.61 & Wiener & 0.68 \\
& & SIR & 0.70 \\
$118.750 \pm 2.100$ & 0.77 & Wiener & 0.78 \\
& & SIR & 0.81 \\
$118.750 \pm 5.000$ & 0.75 & Wiener & 0.76 \\
& & SIR & 0.79 \\
$183.310 \pm 5.000$ & 0.85 & Wiener & 0.82 \\
& & SIR & 0.87 \\
$183.310 \pm 17.000$ & 0.83 & Wiener & 0.81 \\
& & SIR & 0.86 \\
$380.197 \pm 18.000$ & \multirow{2}{*}{0.89} & Bilinear & 0.93 \\
$424.763 \pm 4.000$ & \multirow{3}{*}{0.86} & Interp. \\
& & Bilinear & 0.92 \\
& & Interp. \\
\hline
\end{tabular}

Taking into account that the fundamental objection to embark MW sensors on geostationary platforms is presently related to the large IFOV at GOMAS frequencies, the results we have shown give a more clear idea about the observational capability obtainable after image processing. Considering the enormous advancement that the frequent temporal sampling at MW frequencies can give to precipitation mon- itoring, we consider those results encouraging and the topic worthy to be further investigated.

Acknowledgements. This work has been carried out in the framework of EUMETSAT study contract EUM/CO/04/1386/KJG.

Edited by: V. Kotroni and K. Lagouvardos

Reviewed by: anonymous referee

\section{References}

Bauer, P., Schanz, L., and Roberti, L.: Correction of the threedimensional effects for passive microwave remote sensing of convective clouds, J. Appl. Meteor., 37, 1619-1632, 1998.

Buck, G. J. and Gustincic, J. J.: Resolution Limitations of a Finite Aperture, IEEE Trans. Ant. Prop., 15, 3, 376-381, 1967.

Farrar, M. R. and Smith, E. A.: Spatial resolution enhancement of terrestrial features using deconvolved SSM/I microwave brightness temperatures, IEEE Trans. Geosci. Remote Sens., 30, 349355, 1992.

Jain, A. K.: Fundamentals of Digital Image Processing, PrenticeHall, 1989.

Long, D. G., Hardin, P. J., and Whiting, P. T.: Resolution enhancement of spaceborne scatterometer data, IEEE Trans. Geosci. Remote Sensing, 31, 700-715, 1993.

Long, D. G., Hardin, P. J., and Whiting, P. T.: Resolution enhancement of spaceborne scatterometer data, IEEE Trans. Geosci. Remote Sensing, 36, 407-417, 1998.

Poe, G. A.: Optimum interpolation of imaging microwave radiometer data, IEEE Trans. Geosci. Remote Sens., 28, 800-810, 1990. 
Skou, N.: Microwave Radiometer Systems: Design and Analysis, Artech House Inc., Norwood, hIA, USA, 1989.

Tripoli, G. J.: A nonhydrostatic model designed to simulate scale interaction, Mon. Wea. Rev., 120, 1342-1359, 1992.
Ulaby, F. T., Moore, R. K., and Fung, A. K.: Microwave remote sensing: Active and passive, Vol. 1, Addison-Wesley Publishing Company, Reading, Massachusetts, 1981. 